\title{
Pandemien zeigen, wie sinnvoll die Verbindung unserer Disziplinen ist
}

zurzeit bewegt die Welt die Angst vor einer Pandemie durch die sogenannte Schweinegrippe (besser und richtiger wäre: Mexikanische Grippe). Wenn Sie dies lesen, werden wir sicher schon viel mehr wissen, möglicherweise wird sich bis dahin das ganze Krisenszenario wieder aufgelöst haben. Dafür spricht zumindest der relativ blande Verlauf der Mehrheit der Erkrankungen. Unabhängig davon ist es immer wieder erschreckend, wie schnell sich eine derartige Erkrankung über die Welt verbreiten kann und wie verletzlich wir durch die enge weltweite Vernetzung mit den unglaublich schnellen Verkehrsverbindungen sind.

Diese Erkrankung zeigt auch, wie sinnvoll die Verbindung von Flug-, Reise- und Tropenmedizin ist, da letztendlich all unsere Disziplinen davon betroffen sind, und wie wichtig es ist, sich intensiv damit zu beschäftigen. In dieser Ausgabe der Flugmedizin Tropenmedizin Reisemedizin erfahren Sie in der Rubrik „Magazin“ mehr zu dieser Grippevariante und dem H1N1Virus. Sicher werden wir uns auch weiterhin auf eine Pandemie einstellen müssen - unter Umständen in wesentlich größerem Ausmaß bzw. von größerer Gefährlichkeit. Ein Gedanke, der nicht gerade beruhigend ist, dem wir uns aber letztendlich auf Dauer nicht verschließen können.

Während ich diese Zeilen diktiere, findet noch ein anderes wichtiges Ereignis statt: die diesjährige Tagung der „Aerospace Medical Association" in Los Angeles. Diese weltweit größte Konferenz über Luft- und Raumfahrtmedizin hat nicht nur eine sehr große Tradition, sondern bietet auch eine wunderbare Plattform, mit Kollegen und Experten aus aller Welt über luft- und raumfahrtmedizinische Fragen zu diskutieren. Die Deutsche Gesellschaft für Luftund Raumfahrtmedizin ist seit Jahren ein hochaktives und angesehenes Mitglied dieser Veranstaltung, nicht zuletzt auch durch die deutschsprachige Sitzung, die bisher regelmäßig stattfand.

Es ist bedauerlich, dass sie dieses Jahr in Los Angeles nicht auf dem Programm steht. Hoffentlich wird diese Tradition in den nächsten Jahren wieder aufgenommen - vor allem vor dem Hintergrund, auf welch große Verdienste und Erfolge die Deutsche Luft- und Raumfahrtmedizin sowohl vor als auch nach dem 2 . Weltkrieg zurückblicken kann.

Zuletzt möchte ich auf einen Gedanken von Prof. Rupert Gerzer aus dem letzten Editorial zurückkommen. Die Zeitschrift Flugmedizin Tropenmedizin Reisemedizin hat die Tradition der sehr erfolgreichen Veranstaltung „Medizin und Mobilität“" übernommen, sowohl Flug-, Tropen-, als auch Reisemedizin zusammenzufassen - ich finde, dies ist überaus gut gelungen. Es wäre aber trotzdem wünschenswert, immer wieder einmal über den eigenen Tellerrand zu blicken und uns zu einer gemeinsamen Konferenz zu treffen, wenn vielleicht auch in größeren Abständen.

Ich wünsche Ihnen interessante und kurzweilige Stunden bei der Lektüre dieser Ausgabe der Flugmedizin Tropenmedizin Reisemedizin.

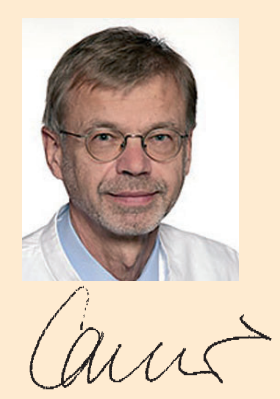

Prof. Dr. Helmut Landgraf, Berlin 